\title{
Genetic variability of sexual size dimorphism in a natural population of Drosophila melanogaster: an isofemale-line approach
}

\author{
JEAN R. DAVID ${ }^{1} *$, PATRICIA GIBERT ${ }^{2}$, SANDRINE MIGNON-GRASTEAU ${ }^{3}$, \\ HÉLÈNE LEGOUT ${ }^{1}$, GEORGES PÉTAVY ${ }^{1}$, CATHERINE BEAUMONT ${ }^{3}$ \\ and BRIGITTE MORETEA U ${ }^{1}$ \\ ${ }^{1}$ CNRS, Laboratoire Populations, Génétique et Evolution, 91198 - Gif sur Yvette Cedex, France \\ ${ }^{2}$ CNRS, Laboratoire Biométrie, Biologie Evolutive, Université de Lyon I 69622 - Villeurbanne Cedex, France \\ ${ }^{3}$ INRA, Station de Recherches Avicoles 37380 - Nouzilly, France
}

\begin{abstract}
Most animal species exhibit sexual size dimorphism (SSD). SSD is a trait difficult to quantify for genetical purposes since it must be simultaneously measured on two kinds of individuals, and it is generally expressed either as a difference or as a ratio between sexes. Here we ask two related questions: What is the best way to describe SSD, and is it possible to conveniently demonstrate its genetic variability in a natural population? We show that a simple experimental design, the isofemale-line technique (full-sib families), may provide an estimate of genetic variability, using the coefficient of intraclass correlation. We consider two SSD indices, the female-male difference and the female/male ratio. For two size-related traits, wing and thorax length, we found that both SSD indices were normally distributed. Within each family, the variability of SSD was estimated by considering individual values in one sex (the female) with respect to the mean value in the other sex (the male). In a homogeneous sample of 30 lines of Drosophila melanogaster, both indices provided similar intraclass correlations, on average 0.21 , significantly greater than zero but lower than those for the traits themselves: 0.50 and 0.36 for wing and thorax length respectively. Wing and thorax length were strongly positively correlated within each sex. SSD indices of wing and thorax length were also positively correlated, but to a lesser degree than for the traits themselves. For comparative evolutionary studies, the ratio between sexes seems a better index of SSD since it avoids scaling effects among populations or species, permits comparisons between different traits, and has an unambiguous biological significance. In the case of D. melanogaster grown at $25^{\circ} \mathrm{C}$, the average female/male ratios are very similar for the wing (1.16) and the thorax (1.15), and indicate that, on average, these size traits are $15-16 \%$ longer in females.
\end{abstract}

[David J. R., Gibert P., Mignon-Grasteau S., Legout H., Pétavy G., Beaumont C. and Moreteau B. 2003 Genetic variability of sexual size dimorphism in a natural population of Drosophila melanogaster: an isofemale-line approach. J. Genet. 82, 79-88]

\section{Introduction}

Most animal species exhibit phenotypic differences between males and females, and after Darwin (1871) sexual size dimorphism (SSD) in particular has retained the attention of numerous evolutionary biologists (Maynard Smith 1978; Charnov 1982; Thornhill and Alcock 1983;

*For correspondence. E-mail: david@pge.cnrs-gif.fr.
Bradbury and Anderson 1987; Stearns 1987; Michod and Levin 1988; Reiss 1989; Andersson 1994; Fairbairn 1997; Simmons 2001). Several adaptive interpretations of SSD have been subject to experimental or theoretical analyses (Slatkin 1984; Arak 1988; Hedrick and Temeles 1989; Kirkpatrick and Ryan 1991).

A general requirement for SSD to evolve is that the trait of interest should be controlled by genes differently expressed in the two sexes. Since the genetic correlations between the sexes are usually high for morphometric

Keywords. sexual size dimorphism; female-male difference; female/male ratio; intraclass correlation; evolvability; isofemale lines. 
traits, theoretical and empirical analyses have predicted a slow evolutionary rate of SSD (Lande 1980; Arnold 1985; Roff 1997; Merilä et al. 1998). In contrast with this expectation, however, field studies have found rapid divergence of phenotypic SSD in geographic populations of the house finch (Badyaev and Hill 2000; Badyaev et al. 2001). Recent investigations in Drosophila have also yielded evidence for different quantitative trait loci (QTL) in males and females for various traits such as bristle number (Mackay 2001), lifespan (Nuzhdin et al. 1997; Leips and Mackay 2000; Vieira et al. 2000) and olfactory behaviour (Mackay et al. 1996).

Although widespread in animals, SSD does not follow a general rule: females can be either larger or smaller than males. Usual adaptive interpretations involve either sexual selection (e.g. competition among males or female choice) or natural selection, assuming divergent ecological advantages. In Drosophila melanogaster, females are larger than males but there is no consensus interpretation for this difference. Larger females have a higher fecundity related to more ovarioles in the ovaries (BoulétreauMerle et al. 1982) and should be favoured by natural selection. Things are not so clear for males. Laboratory experiments have shown that larger males generally have a mating advantage (Partridge et al. 1987; Santos et al. 1988), but not in all conditions (Zamudio et al. 1995). Moreover, such experiments were done with well-fed males, while in nature males must share their time between foraging and mate acquisition. As shown by Blanckenhorn et al. (1995) for a water strider, it is possible that, when resources are limiting, smaller males might be favoured by sexual selection since they require lower amounts of food.

Whatever the evolutionary interpretation of its SSD, D. melanogaster remains an ideal model for genetic investigations. Several studies have analysed SSD changes occurring as a correlated response in various selection regimes, and thus have shown that SSD is genetically variable (e.g. Frankham 1968; Palezona and Alicchio 1973; Cowley et al. 1986; Curtsinger 1986; Reeve and Fairbairn 1996, 1999). We are however aware of only one study (Bird and Schaffer 1972) that directly addressed the heritability of SSD itself in selection experiments. Reeve and Fairbairn (1999) investigated whether a monosexual selection on fecundity could produce a change in SSD as a correlated response, but the results were inconclusive, at least for thorax length.

In Drosophila, the isofemale-line analysis (full-sib families) is a widespread technique for investigating the genetic variability of natural populations (Hoffmann and Parsons 1988; Capy et al. 1994; David et al. 2004) and genotype-environment interactions (David et al. 1994; Karan et al. 1999, 2000). Such investigations have shown that size-related traits are highly heritable, that SSD is a plastic trait, and that female and male sizes are positively correlated. The fact that genetic correlations between males and females were on average close to 0.80 (Karan et al. 1999) suggests that two thirds $\left(R^{2}=0.64\right)$ of the size genes are expressed equally in both sexes. In other words, $36 \%$ of the genes might be expressed specifically in one sex only, leaving significant opportunity for the evolution of SSD. In another study, Reeve and Fairbairn (1996), using a half-sib design, found a still higher value $(r=0.93)$.

SSD may be expressed either as a difference (e.g. female minus male) or as a ratio (e.g. female/male) (Bird and Schaffer 1972; Cowley et al. 1986; Cowley and Atchley 1988; David et al. 1994; Ranta et al. 1994; Reeve and Fairbairn 1996, 1999). The difficulty for calculating heritability is that we need an individual estimate of a trait which must be measured on different individuals.

Up to now, most investigations on SSD have estimated its heritability from selection experiments (e.g. Bird and Shaffer 1972; Reeve and Fairbairn 1996). We present here the results of another method which can be of general use when full-sib data are available (Singh et al. 1989). More precisely, the individual variability in one sex is estimated with respect to the mean value in the other sex. For each isofemale line, we have considered the individual values of the females but only the mean of their brothers. SSD can be expressed either as a difference or as a ratio, and in each case it is possible to calculate a within-family variance.

Using this technique we found that the intraclass correlations of SSD were significantly greater than zero but lower than the intraclass correlations of wing and thorax length. Female-male difference or female/male ratio gave similar intraclass correlations but very different evolvabilities. For comparative purposes, the ratio seems more appropriate than the difference, since it avoids scaling effects and provides an immediate biological interpretation.

\section{Materials and methods}

Flies investigated: We investigated three samples of ten isofemale lines each, collected in the same place (Grande Ferrade estate near Bordeaux, southern France), at the same time of the year (end of autumn) but in different years (1992, 1997, 1999). For the 1992 sample, lines were kept in the lab for four months (four generations) before measurements. It is unlikely that variability among lines was increased owing to genetic drift since, in another study (Gibert et al. 1998a), mean values of wing length remained stable over nine generations. For the 1997 and 1999 samples, measurements were done on the second laboratory generation. Field-collected females were isolated in culture vials to produce laboratory, first-generation progeny $(\mathrm{G} 1)$. Ten pairs of these G1 flies were used as parents for producing the next generation. A 
short egg-laying duration $\left(4 \mathrm{~h}\right.$ at $\left.20-21^{\circ} \mathrm{C}\right)$ was used to limit larval density, which was between 70 and 150 individuals per vial. Moreover, the use of a high-nutrient, killed-yeast medium prevented any visible crowding effect on body size (see Karan et al. 1999). After removal of the parents, vials with eggs were incubated at $25^{\circ} \mathrm{C}$. After emergence from pupae, adults were transferred to fresh food and 10 females and 10 males from each line were measured a few days later. Here we consider only two size-related traits: thorax length measured from the neck to the tip of the scutellum and total wing length, measured, in a left side view, from the thoracic articulation to its extremity. Measurements were made with an ocular micrometer in a binocular microscope with magnification of $25 \times$ for the wing and $50 \times$ for the thorax. Micrometer units were transformed into $\mathrm{mm} \times 100$. We did not investigate the repeatability of length measurements on the same fly. In a previous paper (Imasheva et al. 2000) we found that measurement errors produced a significant increase of variability for small length traits, while they were negligible for longer traits, such as total wing length.

Sexual size dimorphism (SSD): SSD analysis implies separate measurements on both sexes, and two general possibilities exist: the female-male difference (F-M) or the female/male ratio (F/M) (Ranta et al. 1994). With the isofemale-line design, such dimorphism values can be calculated for each line, using the means of males and females (e.g. David et al. 1994). For genetic analysis, we need however a dimorphism value at an individual level. A convenient approach is to consider for one sex (e.g. females) the individual variations $F_{i}$, but for the other sex (males) only the average value $\mathrm{M}_{\mathrm{m}}$. For the ratio we calculate the $F_{i} / M_{m}$, and for the difference the $F_{i}-M_{m}$ values. Such values are individual data providing a mean and a variance. Calculations can be done either on a whole population or also, in our case, separately for each line. Notice that SSD could also be analysed by using male individual variability. In that case, the ratio becomes $\mathrm{F}_{\mathrm{m}} / \mathrm{M}_{\mathrm{i}}$. Relative variabilities (CVs) are identical in males and females
(Karan et al. 1999) and both methods provided similar conclusions. For the sake of simplicity we present here only the $\mathrm{F}_{\mathrm{i}} / \mathrm{M}_{\mathrm{m}}$ data.

\section{Results}

\section{Mean values and sources of variation (ANOVA)}

With the isofemale-line technique, significant variations among lines are regularly observed, reflecting a genetic variability in the wild population (Capy et al. 1994). However, repeated samples from the same population are generally not available. Here we had three such samples, collected in different years, and we first asked two questions: Are there significant differences between samples? Is there a significant interaction between lines and sex? Results for the two traits measured (wing and thorax length) were submitted to a two-way ANOVA (table 1). Sex had the major effect (males are smaller than females). Lines also exhibited, as expected, highly significant genetic variations. There was however no difference between samples collected in different years, and no interaction between year and sex. Finally, a highly significant line by sex interaction means that genetic variability among lines is not identical in males and females. In other words, we may expect that SSD will be genetically variable. The genetic variability among the 30 lines sample is illustrated in figure 1 as a correlation diagram between male and female wing length.

\section{Frequency distributions of traits and SSD indices}

For investigating SSD, we considered two indices: the female/male $(\mathrm{F} / \mathrm{M})$ ratio and the female minus male $(\mathrm{F}-\mathrm{M})$ difference. As stated in Materials and methods, we used in each line the individual female values but the mean value of their brothers.

A general statistical problem when using transformed variables is the shape of the frequency distributions of the new indices. For continuous variables such as wing or thorax length, it is generally assumed that the distribu-

Table 1. Results of a mixed-model ANOVA on wing and thorax length (sex as fixed effect; year as random; line nested in year).

\begin{tabular}{|c|c|c|c|c|c|c|c|}
\hline \multirow[b]{2}{*}{ Source } & \multirow[b]{2}{*}{ d.f. } & \multicolumn{3}{|c|}{ Wing } & \multicolumn{3}{|c|}{ Thorax } \\
\hline & & MS & $F$ & $P$ & MS & $F$ & $P$ \\
\hline Year (1) & 2 & 264.1 & 0.54 & $0.59 \mathrm{~ns}$ & 84.02 & 1.88 & $0.17 \mathrm{~ns}$ \\
\hline Line (year) (2) & 27 & 489.76 & 20.33 & $<0.001$ & 44.67 & 11.16 & $<0.001$ \\
\hline $\operatorname{Sex}(3)$ & 1 & 210413 & 2294 & $<0.001$ & 29962 & 3327 & $<0.001$ \\
\hline $1 * 3$ & 2 & 91.71 & 2.09 & $0.14 \mathrm{~ns}$ & 9.01 & 0.97 & $0.39 \mathrm{~ns}$ \\
\hline $2 * 3$ & 27 & 43.84 & 1.82 & 0.007 & 9.27 & 2.32 & $<0.001$ \\
\hline Error & 540 & 24.09 & & & 4.00 & & \\
\hline
\end{tabular}

d.f., Degree of freedom; MS, mean square; $F$, variance ratio, $P$, probability. 
tions are Gaussian. Such should not be the case for a ratio (e.g. Ranta et al. 1994). We used the whole sample of 300 flies of each sex for analysing the shapes of the distributions of all traits. For wing and thorax length, a preliminary analysis showed no significant difference between sexes in the shape of the distribution. Each distribution was standardized to the same mean (one) by dividing individual values by the overall mean, thus keeping constant the shape and the CV of each distribution. Then, male and female data of each trait were pooled, providing a total sample of 600 observations. The distributions of wing and thorax length were unimodal and symmetrical with no significant skewness or kurtosis. However, the hypothesis of strict normality was rejected in both cases, owing to some irregularities among classes (see figure 2). Such a result is quite surprising and we have no explanation for it. A possibility would be that the bias is due to the fact that the total sample was not drawn from a single panmictic population but made by the addition of 30 independent lines. However, the departure of the distributions from normality is not very big, and ANOVA is known to be a robust technique, so that the conclusions from table 1 are valid.

Result for the SSD indices (F-M and F/M) however failed to show any significant departure from normality. As illustrated in figure 2 for thorax length, the observed distributions were more regular than that of the trait itself. We conclude that either of these indices can be used for describing SSD.

\section{Values of traits and SSD indices in different years}

The mean values of wing and thorax length and of SSD indices of the different samples are given in table 2 . Variations between years for wing and thorax length are

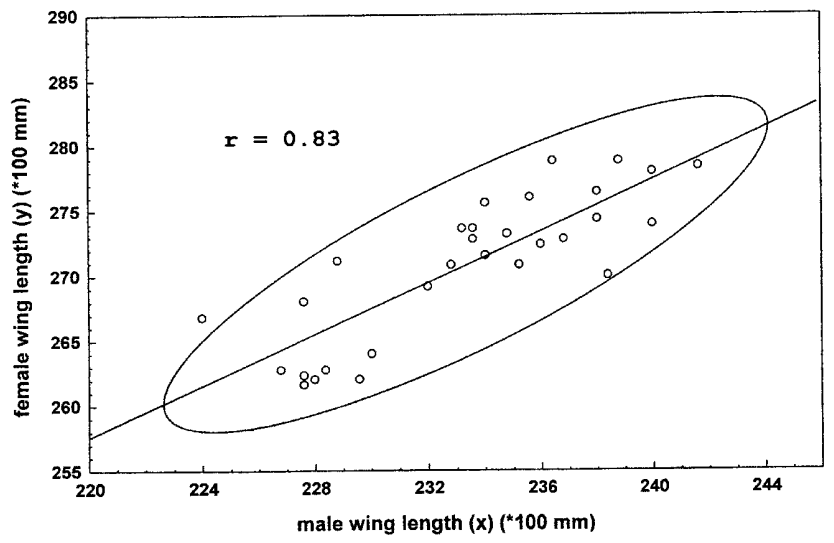

Figure 1. Correlation diagram between male and female wing length. Each value is the mean of an isofemale line. Ellipse of $90 \%$ probability is given to help visualize the overall distribution. The regression line is a type 1 regression, female as a function of male: $F=39.80+0.99 \mathrm{M}+\varepsilon$. This kind of regression is implicit when calculating a female/male ratio. small and nonsignificant, suggesting that the population sampled was stable for these traits over time. The same conclusion applies to SSD indices. They are not different between years but significantly variable among lines. Interestingly, we notice that the variance ratios ( $F$ parameters) are always superior for wing and thorax length than for SSD. This suggests that SSD indices are less variable among lines than the traits themselves.

For the French population investigated, the F-M difference is $37.45 \pm 1.25$ for the wing but only $14.13 \pm 0.52$
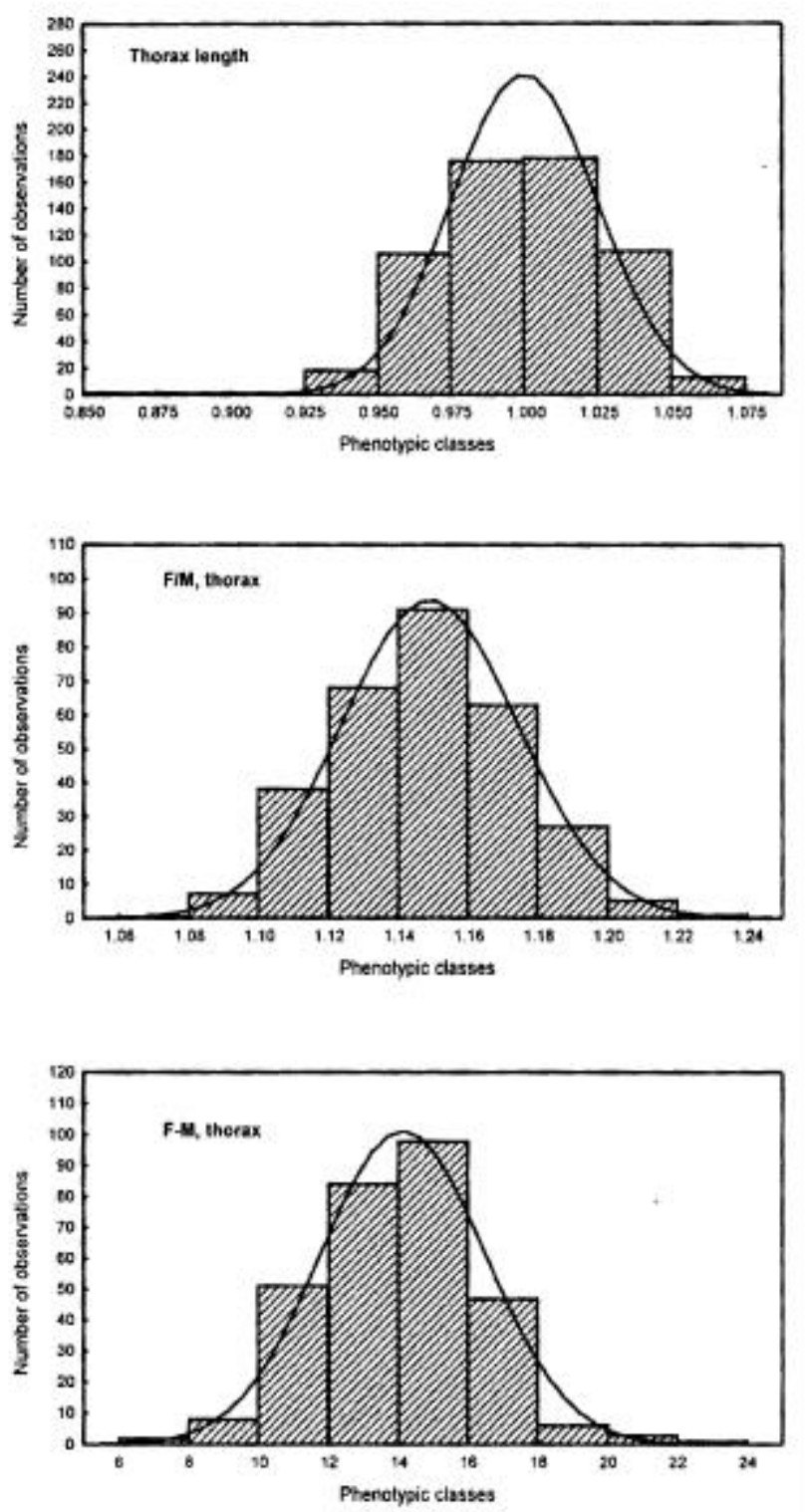

Figure 2. Frequency distributions of thorax length and SSD indices $\mathrm{F} / \mathrm{M}$ ratio and $\mathrm{F}-\mathrm{M}$ difference based on the total sample. Experimental histograms are compared with the corresponding normal distributions. Normality was rejected by statistical tests (Kolmogorov-Smirnov, Lilliefors and ShapiroWilk $W$ ) for thorax length, but accepted for F/M or F-M. 
for the thorax. The dimorphism, expressed in that way, appears much greater for the wing. This is a mere consequence of the fact that in Drosophila the wing is much longer than the thorax. When dimorphism is expressed as a ratio, the difference between the two traits almost disappears, since the mean values are $1.161 \pm 0.005$ and $1.149 \pm 0.006$ for wing and thorax length respectively. This very small difference is however significant: Student's test $t=3.06 ; P=0.003$; paired data. For a given trait, the F-M and the F/M indices calculated for each line are highly correlated $(r>0.95)$, suggesting that they provide basically the same information. This relationship is illustrated in figure 3 for the wing. From a biological point of view, the SSD ratio might be preferred, since it tells that female traits are on average 15-16\% longer than those of males.

\section{Genetic analyses}

Using standard methods, we calculated (table 3) for each sample the intraclass correlation $t$, sometimes called isofemale-line heritability (Capy et al. 1994) and the genetic CV, also called evolvability (Houle 1992).

For wing and thorax length, we found high intraclass correlation, greater for the wing $(0.50 \pm 0.03)$ than for the thorax $(0.37 \pm 0.03)$. The difference among paired data ( $d=0.130 \pm 0.028, n=6, P<0.01)$ is highly significant.

SSD indices exhibited regularly lower values of $t$. Mean values were $0.188 \pm 0.034$ and $0.243 \pm 0.049$ for wing and thorax length respectively ( $n=6$ in each case). For the two index types, means were $0.225 \pm 0.047$ and $0.207 \pm 0.041$, for the ratio and the difference respectively $(n=6)$. ANOVA (not shown) applied to these data did not reveal any significant effect either due to trait or to index type. Moreover the $t$ values for the ratio and the difference are highly and positively correlated $(r=0.98$, $n=6$ ), again suggesting that both indices provide the same genetic information. For a given sample and trait, the best estimate of $t$ seems to be the average value found for the two indices, the mean of which is $0.216 \pm 0.044$ $(n=6)$. When intraclass correlations of SSD and of trait values are compared, they appear however to be independent. Indeed we found (figure 4) a negative, although nonsignificant, correlation between them.

Evolvability $(\mathrm{CVg})$ was slightly superior for wing length $(m=1.94 \pm 0.07, n=6)$ than for thorax length ( $m=1.48 \pm 0.03, n=6$ ). The average difference of paired data is highly significant $(d=0.455 \pm 0.088, n=6$, $P<0.001$ ). Evolvability was still less (average $1.02 \pm$ $0.17, n=6$ ) for the $\mathrm{F} / \mathrm{M}$ ratio, but, quite surprisingly, much greater for the F-M difference (average $7.12 \pm$ $1.04, n=6)$.

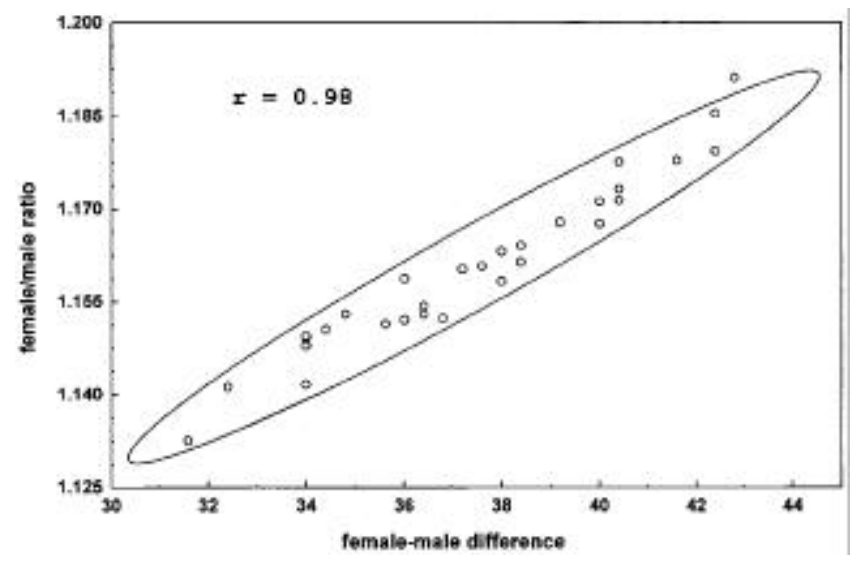

Figure 3. Correlation diagram between two sexual dimorphism indices of wing length among isofemale lines.

Table 2. Mean values of size traits and SSD indices and results of a one-way ANOVA (lines nested in samples).

\begin{tabular}{|c|c|c|c|c|c|c|c|c|c|}
\hline & & \multicolumn{2}{|c|}{ Wing length } & \multicolumn{2}{|c|}{ Thorax length } & \multicolumn{2}{|c|}{ SSD ratio } & \multicolumn{2}{|c|}{ SSD difference } \\
\hline & & Female & Male & Female & Male & Wing & Thorax & Wing & Thorax \\
\hline & $\begin{array}{c}1992 \\
(n=10)\end{array}$ & $270.5 \pm 4.55$ & $234.5 \pm 3.97$ & $108.6 \pm 1.77$ & $95.0 \pm 1.76$ & $1.154 \pm 0.019$ & $1.144 \pm 0.019$ & $36.00 \pm 4.55$ & $13.66 \pm 1.77$ \\
\hline \multirow[t]{2}{*}{$\begin{array}{l}\text { Mean } \\
\text { values }\end{array}$} & $\begin{array}{c}1997 \\
(n=10)\end{array}$ & $269.6 \pm 4.86$ & $232.0 \pm 4.79$ & $108.6 \pm 1.95$ & $94.2 \pm 1.79$ & $1.162 \pm 0.021$ & $1.154 \pm 0.021$ & $37.68 \pm 4.86$ & $14.48 \pm 1.95$ \\
\hline & $\begin{array}{c}1999 \\
(n=10)\end{array}$ & $272.3 \pm 5.18$ & $233.6 \pm 5.03$ & $109.8 \pm 2.25$ & $95.5 \pm 2.07$ & $1.166 \pm 0.022$ & $1.149 \pm 0.024$ & $38.68 \pm 5.18$ & $14.26 \pm 2.25$ \\
\hline $\begin{array}{l}\text { Overall } \\
\text { mean }\end{array}$ & $(n=30)$ & $270.8 \pm 1.25$ & $233.4 \pm 1.40$ & $109.0 \pm 0.52$ & $94.9 \pm 0.54$ & $1.161 \pm 0.0054$ & $1.149 \pm 0.0055$ & $37.45 \pm 1.25$ & $14.13 \pm 0.519$ \\
\hline \multicolumn{10}{|c|}{ Comparison (ANOVA) } \\
\hline Samples & $F(2,27)$ & $0.59^{\mathrm{ns}}$ & $0.77^{\mathrm{ns}}$ & $1.49^{\mathrm{ns}}$ & $2.01^{\mathrm{ns}}$ & $2.34^{\mathrm{ns}}$ & $1.07^{\mathrm{ns}}$ & $2.09^{\mathrm{ns}}$ & $0.97^{\mathrm{ns}}$ \\
\hline Lines & $F(27,270)$ & $12.51 * * *$ & $9.51 * * *$ & $7.13 * * *$ & $6.30 * * *$ & $3.70 * * *$ & $5.05 * * *$ & $3.49 * * *$ & $4.41 * * *$ \\
\hline
\end{tabular}

Mean values are the mean of $n$ lines. Degrees of freedom are given for ANOVA: MS, mean square; $F$, variance ratio. SSD ratio, sexual dimorphism expressed as a female/male ratio; SSD difference; sexual dimorphism expressed as a female-male difference.

Significance level: ns, nonsignificant; $* * * P<0.001$. 
Jean R. David et al.

Table 3. Values of genetic parameters for size traits and SSD in different samples.

\begin{tabular}{|c|c|c|c|c|c|c|c|c|c|c|c|c|c|c|c|c|}
\hline \multirow[b]{3}{*}{ Sample } & \multicolumn{4}{|c|}{ Wing length } & \multicolumn{4}{|c|}{ Thorax length } & \multicolumn{4}{|c|}{ SSD ratio } & \multicolumn{4}{|c|}{ SSD difference } \\
\hline & \multicolumn{2}{|c|}{ Female } & \multicolumn{2}{|c|}{ Male } & \multicolumn{2}{|c|}{ Female } & \multicolumn{2}{|c|}{ Male } & \multicolumn{2}{|c|}{ Wing } & \multicolumn{2}{|c|}{ Thorax } & \multicolumn{2}{|c|}{ Wing } & \multicolumn{2}{|c|}{ Thorax } \\
\hline & $\mathrm{CVg}$ & $t$ & $\mathrm{CVg}$ & $t$ & $\mathrm{CVg}$ & $t$ & $\mathrm{CVg}$ & $t$ & $\mathrm{CVg}$ & $t$ & $\mathrm{CVg}$ & $t$ & $\mathrm{CVg}$ & $t$ & $\mathrm{CVg}$ & $t$ \\
\hline 1992 & 2.06 & 0.59 & 1.87 & 0.53 & 1.49 & 0.45 & 1.55 & 0.40 & 0.65 & 0.13 & 0.59 & 0.11 & 4.88 & 0.13 & 4.16 & 0.09 \\
\hline 1997 & 1.99 & 0.53 & 1.66 & 0.38 & 1.55 & 0.40 & 1.54 & 0.38 & 0.73 & 0.13 & 1.49 & 0.38 & 5.64 & 0.15 & 10.32 & 0.35 \\
\hline 1999 & 1.91 & 0.49 & 2.13 & 0.47 & 1.37 & 0.30 & 1.39 & 0.28 & 1.35 & 0.32 & 1.32 & 0.28 & 8.44 & 0.27 & 9.28 & 0.25 \\
\hline Mean & 1.99 & 0.54 & 1.89 & 0.46 & 1.47 & 0.38 & 1.49 & 0.35 & 0.91 & 0.19 & 1.13 & 0.26 & 6.32 & 0.18 & 7.92 & 0.23 \\
\hline \pm S.E. & 0.04 & 0.03 & 0.14 & 0.04 & 0.05 & 0.04 & 0.05 & 0.04 & 0.22 & 0.06 & 0.28 & 0.08 & 1.08 & 0.04 & 1.90 & 0.08 \\
\hline
\end{tabular}

$\mathrm{CVg}$, Genetic coefficient of variation (evolvability); $t$, intraclass correlation.

Genetic correlations between traits were estimated using the 30 family means (see Via 1984; Gibert et al. 1998b). Correlations between wing and thorax were high (figure $5 \mathrm{~A})$, identical in both sexes $(r=0.78)$, and slightly greater than the within-line average values, $0.680 \pm 0.042$ and $0.708 \pm 0.034(n=30)$, for males and females respectively. For the SSD indices, we also found a positive correlation between the two traits, but slightly less than for the traits themselves: $r$ of 0.66 and 0.68 for the ratio and the difference respectively. SSD values of wing were also positively correlated to those of thorax (figure 5B), $r$ of 0.66 and 0.68 for the ratio and the difference respectively. This suggests that about $45 \%$ of the genes responsible for SSD are expressed in a similar way in wing and thorax.

\section{Discussion and conclusions}

Although SSD is an important phenomenon in evolutionary biology, few investigations have been devoted to the analysis of its genetic variability. Sophisticated statistical methods such as REML (restricted maximum likelihood) allow the treatment of male and female traits as different and thus permit calculation of individual values of dimorphism (Mignon-Grasteau et al. 1998). We show here that the simple and widespread method of isofemale lines may also be used to assess genetic variation of SSD.

The isofemale-line technique here implemented is a full-sib design which can be used to investigate the genetic architecture of a natural population of various species, provided they can be reared under identical conditions (David et al. 2004). It is convenient for estimating the mean of a quantitative trait in a natural population and it also provides some information about its genetic variability. This last point however raises some difficulties. According to classical quantitative-genetic analysis (Falconer 1989) intraclass correlation $t$ among full sibs should be half the heritability of the trait. This relationship, however, assumes that the genetic variance is purely additive. Empirical observations have shown that $t$ is generally closer to $h^{2}$ than to $0.5 h^{2}$. For example, in Drosophila, a compilation of heritability values obtained with usual methods

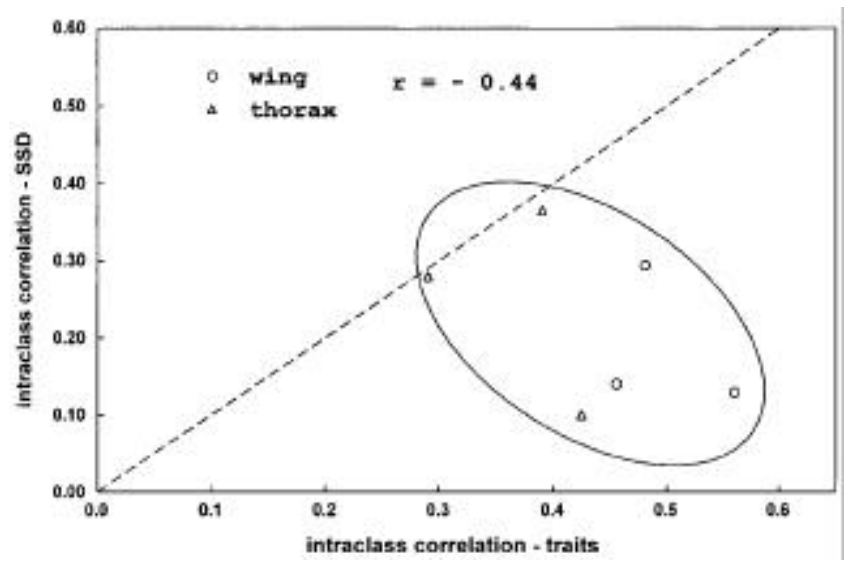

Figure 4. Relationship between intraclass correlation of trait length (wing or thorax) and intraclass correlation of SSD. Sexes were averaged for trait values; ratio and difference indices were averaged for SSD. Mean values: traits, $0.433 \pm 0.037$; SSD indices, $0.215 \pm 0.043$; average difference (paired data): $0.218 \pm$ $0.68(n=6, P<0.01)$.

(parent-offspring regression, half-sib analysis or directional selection) was done by Roff and Mousseau (1987). They found average heritabilities of $0.325 \pm 0.014(n=66)$ and $0.319 \pm 0.021(n=30)$ for wing and thorax length respectively. Using the isofemale-line technique, Capy et al. (1994) found average intraclass correlations of $0.403 \pm 0.021(n=55)$ and $0.232 \pm 0.019(n=55)$ for the same traits respectively. Clearly $t$ (intraclass correlation) is intermediate between $h^{2}$ and $0.5 h^{2}$.

There are two possible explanations for this result: either the variance among lines is overestimated, because of common environment effects, or the genetic variance, among lines, is not purely additive, and includes significant nonadditive (dominance and epistasis) components, and also potential maternal effects.

We extensively investigated the common environment hypothesis and came to the conclusion that it is of minor importance. For example we used two different culture vials for rearing the same family and never found a significant vial effect (J. R. David, unpublished data). Acci- 
dental effects among culture vials should also produce erratic variations when successive generations of the same line are measured. A detailed analysis (Gibert et al. 1998a) revealed this was not the case: mean wing length values of successive generations were strongly correlated, revealing a good genetic repeatability. We also investigated the possible influence of larval density upon size trait values, and failed to find any effect for densities ranging between 20 and 320 eggs per vial (Karan et al. 1999). It is worth emphasizing that such stable results are obtained when using a special rearing medium in which a large amount of dead yeast decreases the environmental variance (David 1962). For example, with that kind of food, the within-line CVs are usually less than $2 \%$ (Gibert et al. 1998a). Such is not the case in other investigations which used a more diluted food. For example, Imasheva et al. (1994) reported an average within-line $\mathrm{CV}$ of $2.95 \pm 0.15$. It is possible that an accidental increase of the within-line variance may reduce the intraclass correlation. Indeed, Hoffmann and Parsons (1988) reported a very low intraclass correlation $(t=0.04 \pm 0.03)$ for wing length, something we never found with our rearing method.
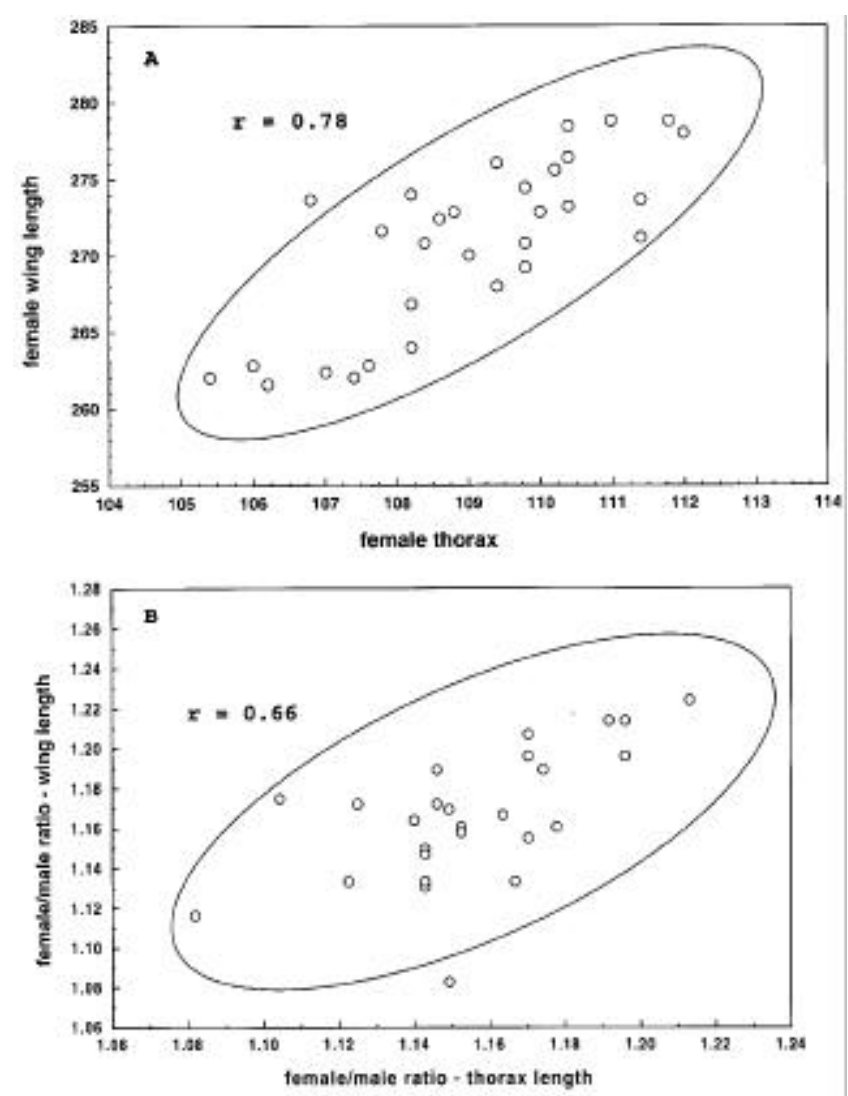

Figure 5. Correlation among family means: A, between wing and thorax lengths in female; B, between SSD ratios of wing and thorax. Ellipses of $90 \%$ probability are shown to better visualize the shapes of distributions.
For all these reasons we consider that intraclass correlation in an isofemale-line design provides a gross measure of genetic variation, including nonadditive components and also possible maternal effects. Other investigators (e.g. Ritchie and Kyriacou 1994) also drew attention to the fact that a behavioural trait, which did not respond to directional selection, indicating lack of additive genetic variation, did nevertheless show significant genetic variation among isofemale lines.

SSD is generally expressed either as a difference or as a ratio, and both have drawbacks. The difference between the sexes $(\mathrm{F}-\mathrm{M})$ is likely to be strongly influenced by absolute differences in body size, owing to allometric relationships (Reiss 1989; Fairbairn 1997), especially when different species are compared. Also, the difference will exhibit large heterogeneity among size traits, as shown here for wing and thorax length. To obviate this difficulty, relative differences standardized to mean size might be used (Ranta et al. 1994). An SSD ratio (F/M) is such a relative measure, the meaning of which is easily understood. Moreover, it allows direct comparison among different traits: in Drosophila SSD ratios are almost identical for wing and thorax length. Ratios often exhibit some inconveniences, one being an expected non-normal distribution (LaBarbera 1989; Ranta et al. 1994; Sokal and Rohlf 1995). Perhaps surprisingly, in our data set the shape of the wing and thorax length distributions departed slightly but significantly from normality, whereas that of the two SSD indices did not. Although we do not have any clear explanation for this result we may conclude that, in the present case, there is no statistical objection against the use of the ratio. A classical way to remedy the expected non-normal distribution of a ratio is a logarithmic transformation, since $\log \mathrm{F} / \mathrm{M}=\log \mathrm{F}-\log$ M. We did this and found that the difference between logs was also normally distributed. We think, however, that the real ratio provides a more straightforward biological interpretation: female wing length is $16 \%$ longer than that of the male. Whether normality of SSD indices holds for other data sets needs to be evaluated further.

The two estimates of SSD (F/M ratio and F-M difference) provided significant intraclass correlations (on average $0.215 \pm 0.018, n=4$ ). As one might expect, they were smaller than for each size trait. This value can be compared to the average realized heritabilities of SSD found by Bird and Schaffer (1972) and Reeve and Fairbairn (1996): respectively, 0.15 and 0.13. Log transformations produced similar estimates. A smaller value of heritability of SSD than for the traits themselves seems a general feature in animals (Mignon-Grasteau et al. 1998). Our results for Drosophila also confirm data obtained with an REML technique on chicken and duck (MignonGrasteau et al. 1998), showing that both SSD indices provide similar heritabilities. We therefore suggest that 
our method could and should be more widely applied, for example in half-sib or diallel designs.

The much higher evolvability found for the F-M difference than for the ratio must be considered with caution, since it might be due to a scaling effect (see Roff 1997, p. 122). Such scaling effects were described for sex dimorphism in bristle number by Frankham (1968). In most genetic investigations so far performed on SSD, either in Drosophila (Bird and Schaffer 1972), mice (Korkman 1957; Eisen and Hanrahan 1972; Schmidt 1993) or birds (Buvanendran 1969; Pilla 1974; Singh et al. 1989; Badyaev et al. 2001), the F-M difference was preferentially considered. This might be related to the higher evolvability found for this SSD index: any change due to selection should be more visible on the F-M difference than on the ratio. In domestic birds, directional selection has been performed for increasing body weight. Owing to a scaling effect, such selection has often increased the F-M difference without really changing the allometric relationship between sexes (see MignonGrasteau et al. 1999). In this respect, the $\mathrm{F} / \mathrm{M}$ ratio in adults is likely to be more informative concerning the evolution of SSD itself.

Using an SSD ratio (thorax width), Reeve and Fairbairn (1996) found several discrepancies between expected and realized heritabilities. One possible explanation was that various selection regimes resulted in different modifications of growth trajectories in males and females. Clearly, more precise investigations are needed for understanding the significance and genetic bases of SSD indices.

When comparing different species, major changes in SSD may be found. For example, in the drosophilid family, males and females may be about the same size (Kacmarczyk and Craddock 2000; Karan et al. 2000). It would be interesting to know if, in such cases, SSD is still genetically variable.

Sexually dimorphic characters imply a functional interaction between the genes responsible for the sexual phenotype and the genes determining the trait of interest. In this respect, sexual dimorphism of morphological traits may be analysed not only by investigating the mean values of male and female, plus the associated genetic correlation, but also by comparing the heritabilities in the two sexes (Cowley et al. 1986; Cowley and Atchley 1988; Reeve and Fairbairn 1996): a significant difference is likely to favour the evolution of SSD. Although females are on average larger than males in D. melanogaster, the $\mathrm{F} / \mathrm{M}$ ratio may be quite variable depending on the trait measured, from 1.04 up to 1.28 (Cowley and Atchley 1988; Reeve and Fairbairn 1999). Other traits not related to size are also sexually dimorphic and convenient for analysing sex-trait interactions. Such interactions play a major role in the control of body pigmentation (Gibert et al. 1999; Hollocher et al. 2000). Indeed, the interacting genes producing the black pigmentation of the male abdomen of $D$. melanogaster have been identified recently (Kopp et al. 2000). Other quantitative investigations in Drosophila, using a QTL approach, have also demonstrated different QTLs in males and females (Mackay et al. 1996; Nuzhdin et al. 1997; Leips and Mackay 2000; Vieira et al. 2000; Mackay 2001). Also, a DNA microarray analysis has revealed different gene expressions in sexes and, more interestingly, different interactions in two sibling Drosophila species (Ranz et al. 2003). Altogether, the genetics of sexual dimorphism might become a paradigm for the analysis of epistatic interactions, a field of growing significance in quantitative evolutionary genetics (Wolf et al. 2000)

\section{Acknowledgements}

We thank Drs W. U. Blanckenhorn and T. F. Mackay for helpful comments on an earlier draft of this paper.

\section{References}

Andersson M. 1994 Sexual selection. Princeton University Press, Princeton.

Arak A. 1988 Sexual dimorphism in body size: a model and a test. Evolution 42, 820-825.

Arnold S. J. 1985 Quantitative genetic models of sexual selection. Evolution 41, 1296-1310.

Badyaev A. V. and Hill G. E. 2000 The evolution of sexual dimorphism in the house finch. I. Population divergence in morphological covariance structure. Evolution 54, 17841794.

Badyaev A. V., Hill G. E. and. Whittingham L. A. 2001 The evolution of sexual size dimorphism in the house finch. IV. Population divergence in ontogeny. Evolution 55, 25342549.

Bird M. A. and Schaffer H. E. 1972 A study of the genetic basis of the sexual dimorphism for wing length in Drosophila melanogaster. Genetics 72, 475-487.

Blanckenhorn W. U., Preziosi R. F. and Fairbairn D. J. 1995 Time and energy constraints and the evolution of sexual size dimorphism - to eat or to mate? Evol. Ecol. 9, 369-381.

Boulétreau-Merle J., Allemand R., Cohet Y. and David J. R. 1982 Reproductive strategy in Drosophila melanogaster: significance of a genetic divergence between temperate and tropical populations. Oecologia 53, 323-329.

Bradbury J. W. and Anderson M. B. 1987 Sexual selection: testing the alternatives. Wiley, Chichester.

Buvanendran V. 1969 The heritability and genetic correlations of sexual dimorphism for 10 -week weight in poultry. $\mathrm{Br}$. Poult. Sci. 10, 321-325.

Capy P., Pla E. and David J. R. 1994 Phenotypic and genetic variability of morphometrical traits in natural populations of Drosophila melanogaster and D. simulans. II. Withinpopulation variability. Genet. Sel. Evol. 26, 15-28.

Charnov E. L. 1982 The theory of sex allocation. Princeton University Press, Princeton.

Cowley D. E. and Atchley W. R. 1988 Quantitative genetics of Drosophila melanogaster II. Heritabilities and genetic correlations between sexes for head and thorax traits. Genetics 119, 421-433. 


\section{Sexual size dimorphism in Drosophila}

Cowley D. E, Atchley W. R. and Rutledge J. J. 1986 Quantitative genetics of Drosophila melanogaster I. sexual dimorphism in genetics parameters for wing traits. Genetics 114, $549-566$

Curtsinger J. W. 1986 Quantitative wing variation in inbred and outbred lines of Drosophila melanogaster. J. Hered. 77, 267-271.

Darwin C. 1871 The descent of man and selection in relation to sex. John Murray, London.

David J. 1962 A new medium for rearing Drosophila in axenic conditions. Drosoph. Inf. Serv. 36, 128.

David J. R., Moreteau B., Gautier J. P., Pétavy G., Stockel J. and Imasheva A. 1994 Reaction norms of size characters in relation to growth temperature in Drosophila melanogaster: an isofemale-lines analysis. Genet. Sel. Evol. 26, 229-251.

David J. R., Gibert P., Legout H., Pétavy G., Capy P. and Moreteau B. 2004 Isofemale lines in Drosophila: an empirical approach to quantitative trait analysis in natural populations. Heredity (in press).

Eisen E. and Hanrahan J. P. 1972 Selection for sexual dimorphism in body weight in mice. Aust. J. Biol. Sci. 25, 10151024.

Fairbairn D. J. 1997 Allometry for sexual size dimorphism: pattern and process in the coevolution of body size in males and females. Annu. Rev. Ecol. Syst. 28, 659-687.

Falconer D. S. 1989 Introduction to quantitative genetics. Longman, New York.

Frankham R. 1968 Sex and selection for a quantitative trait in Drosophila. II. The sex dimorphism. Aust. J. Biol. Sci. 21, 1215-1223.

Gibert P., Moreteau B., Moreteau J. C. and David J. R. 1998a Genetic variability of quantitative traits in Drosophila melanogaster (fruit fly) natural populations: analysis of wild living flies and of several laboratory generations. Heredity 80, 326-335.

Gibert P., Moreteau B., Scheiner S. M. and David J. R. 1998b Phenotypic plasticity of body pigmentation in Drosophila: correlated variations between segments. Genet. Sel. Evol. 30, 181-194.

Gibert P., Moreteau B., Munjal A. K. and David J. R. 1999 Phenotypic plasticity of abdominal pigmentation in Drosophila kikkawai: multiple interactions between a major gene, sex, abdomen segment and growth temperature. Genetica 105, $165-176$

Hedrick A. V. and Temeles E. J. 1989 The evolution of sexual dimorphism in animals: hypotheses and tests. Trends Ecol. Evol. 4, 136-138.

Hoffmann A. A. and Parsons P. A. 1988 The analysis of quantitative variation in natural populations with isofemale strains. Genet. Sel. Evol. 20, 87-98.

Hollocher H., Hatcher J. L. and Dyreson E. G. 2000 Evolution of abdominal pigmentation differences across species in the Drosophila dunni subgroup. Evolution 54, 2046-2056.

Houle D. 1992 Comparing evolvability and variability of quantitative traits. Genetics 130, 195-204.

Imasheva A. G., Bubli O. A. and Lazebny O. E. 1994 Variation in wing length in Eurasian populations of Drosophila melanogaster. Heredity 72, 508-514.

Imasheva A. G., Moreteau B. and David J. R. 2000 Growth temperature and genetic variability of wing dimensions in Drosophila: opposite trends in two sibling species. Genet. Res. 76, 237-247.

Kacmarczyk T. and Craddock E. M. 2000 Cell size is a factor in body size variation among Hawaiian and non-Hawaiian species. Drosoph. Inf. Serv. 83, 144-148.
Karan D., Morin J. P., Gravot E., Moreteau B. and David J. R. 1999 Body size reaction norms in Drosophila melanogaster: temporal stability and genetic architecture in a natural population. Genet. Sel. Evol. 31, 491-508.

Karan D., Dubey S., Moreteau B., Parkash R. and David J. R. 2000 Geographical clines for quantitative traits in natural populations of a tropical drosophilid: Zaprionus indianus. Genetica 108, 91-100.

Kirkpatrick M. and Ryan M. J. 1991 The evolution of mating preferences and the paradox of the lek. Nature 350, 3338.

Kopp A., Duncan I. and Carroll S. B. 2000 Genetic control and evolution of sexually dimorphic characters in Drosophila. Nature 408, 553-559.

Korkman N. 1957 Selection with regard to the sex difference of body weight in mice. Hereditas 43, 665-678.

LaBarbera M. 1989 Analyzing body size as a factor in ecology and evolution. Annu. Rev. Ecol. Syst. 20, 97-117.

Lande R. 1980 Sexual dimorphism, sexual selection and adaptation in phylogenic characters. Evolution 34, 293-305.

Leips J. and Mackay T. F. 2000 Quantitative trait loci for life span in Drosophila melanogaster: interactions with genetic background and larval density. Genetics 155, 1773-1788.

Mackay T. F. C. 2001 The genetic architecture of quantitative traits. Annu. Rev. Genet. 35, 303-339.

Mackay T. F. C., Hackett J. B., Lyman R. F., Wayne M. L. and Anholt R. R. H. 1996 Quantitative genetic variation of odorguided behaviour in a natural population of Drosophila melanogaster. Genetics 144, 727-735.

Maynard Smith J. 1978 The evolution of sex. Cambridge University Press, Cambridge.

Merilä J., Sheldon B. C and Ellegren H. 1998 Quantitative genetics of sexual size dimorphism in the collared flycatcher, Ficedula albicollis. Evolution 52, 870-880.

Michod R. E. and Levin B. R. 1988 The evolution of sex. Sinauer, Sunderland.

Mignon-Grasteau S., Beaumont C., Poivey J. P. and De Rochambeau H. 1998 Estimation of the genetic parameters of sexual dimorphism of body weight in "label" chicken and muscovy ducks. Genet. Sel. Evol. 30, 481-491.

Mignon-Grasteau S., Beaumont C., Le Bihan-Duval E., Poivey J. P., De Rochambeau H. and Ricard F. H. 1999 Genetic parameters of growth curve parameters in male and female chickens. Br. Poult. Sci. 40, 44-51.

Nuzhdin S. V., Pasyukova E. G., Dilda C. L., Zeng Z. B. and Mackay T. F. 1997 Sex-specific quantitative trait loci affecting longevity in Drosophila melanogaster. Proc. Natl. Acad. Sci. USA 94, 9734-9739.

Palezona D. L. and Alicchio R. 1973 Genetic analysis of sexual dimorphism in Drosophila melanogaster. Monitore Zool. Ital. 4, 63-70.

Partridge L., Ewing A. and Chandler A. 1987 Male size and mating success in Drosophila melanogaster: the roles of male and female behavior. Anim. Behav. 35, 555-562.

Pilla A. M. 1974 Possibilita di miglioramento genetico dell'anatra muschiata. Ann. Inst. Sper. Zootec. 7, 165-174.

Ranta E., Laurila A. and Elmberg J. 1994 Reinventing the wheel: analysis of sexual dimorphism in body size. Oikos 70, 313321.

Ranz J. M., Castillo-Davis C. I., Meiklejohn C. D. and Hartl D. L. 2003 Sex-dependent gene expression and evolution of the Drosophila transcriptome. Science 300, 1742-1745.

Reeve J. P. and Fairbairn D. J. 1996 Sexual size dimorphism as a correlated response to selection on body size: an empirical test of the quantitative genetic model. Evolution 50, 19271938 . 
Reeve J. P. and Fairbairn D. J. 1999 Change in sexual size dimorphism as a correlated response to selection on fecundity. Heredity 83, 697-706.

Reiss M. J. 1989 The allometry of growth and form. Cambridge University Press, Cambridge.

Ritchie M. G. and Kyriacou C. P. 1994 Genetic variability of courtship song in a population of Drosophila melanogaster. Anim. Behav. 48, 425-434.

Roff D. A. 1997 Evolutionary quantitative genetics. Chapman and Hall, London.

Roff D. A. and Mousseau T. A. 1987 Quantitative genetics and fitness: lessons from Drosophila. Heredity 58, 103-118.

Santos M., Ruiz A., Barbadilla A., Quezada-Diaz J. E., Hasson E. and Fontdevila A. 1988 The evolutionary history of Drosophila buzzatii. Heredity 61, 255-262.

Schmidt T. A. 1993 Divergent selection for sexual dimorphism in mice -6 generations of selection. Proceedings of the 44th Annual Meeting EAAP, Aarhus.

Simmons L. W. 2001 Sperm competition and its evolutionary consequences in the insects. Princeton University Press, Princeton.

Singh S. S., Verma S. K., Khan A. G. and Shrivastava A. K. 1989 Studies on genetic variability in juvenile body weights and sexual dimorphism in layer type chicken. Indian J. Poult. Sci. 24, 308-310.

Slatkin M. 1984 Ecological causes of sexual dimorphism. Evolution 38, 622-630.

Sokal R. R. and Rohlf F. J. 1995 Biometry, 3rd edition. Freeman, New York.

Stearns S. C. (ed.) 1987 The evolution of sex and its consequences. Birkhäuser, Basel.

Thornhill R. and Alcock J. 1983 The evolution of insect mating systems. Harvard University Press, Cambridge.

Via S. 1984 The quantitative genetics of polyphagy in an insect herbivore. II. Genetic correlations in larval performance within and among host plants. Evolution 38, 896-905.

Vieira C., Pasyukova E. G., Zeng Z. B., Hackett J. B., Lyman R. F. and Mackay T. F. 2000 Genotype-environment interaction for quantitative trait loci affecting life span in Drosophila melanogaster. Genetics 154, 213-227.

Wolf J. B., Brodie E. D. and Wade M. J. 2000 Epistasis and the evolutionary process. Oxford University Press, New York.

Zamudio K. R., Huey R. B. and Crill W. D. 1995 Bigger isn't always better: body size, developmental and parental temperature and male territorial success in Drosophila melanogaster. Anim. Behav. 49, 671-677. 